A $4 \%$ chlorhexidine solution is not comercially available we contracted Galantic Pharma to prepare supplies for two large clinical trials in Africa. This study aimed to evaluate the effect this $4 \%$ chlorhexidine solution on the colonization and colony counts in hospital born infants.

Methods Newborns ( $n=247$ ) from normal deliveries at a hospital in New Delhi were randomly assigned to one of three groups chlorhexidine, placebo or dry cord care. Swab samples were used to collect smear samples before, after $2 \mathrm{hrs}$ and $48 \mathrm{hrs}$ of application of chlorhexidine and at same times from the dry cord group. All swabs were analysed for growth and colony counts.

Results The overall baseline positivity was 20\% (50 of 247 swabs). Chlorhexidine reduced colonisation and bacterial counts in both ( $2 \mathrm{hr}$ and $48 \mathrm{hr}$ ) samples. As compared to placebo and dry cord, the reduction in positivity in chlorhexidine group in 2-hour samples was $80 \%$ [odds ratio of $0.20, p=0.001$ and odds of $0.19, p=0.00$ respectively]. In 48-hour post intervention, chlorhexidine significantly reduced colonisation in comparison to placebo [difference in mean of $-1.01, p=0.006$ ] and dry cord [difference in mean -1.16 , $\mathrm{p}=0.004]$.

Conclusion Cord cleaning with 4\% Chlorhexidine soon after birth reduces colonisation as well as density of pathogens significantly.

\section{VITAMIN D SUPPLEMENTATION AND THE RISK OF INFECTIONS IN FULLTERM INFANTS. CORRELATIONS WITH THE MATERNAL SERUM VITAMIN D}

doi:10.1136/archdischild-2012-302724.0896

AAM El-Mazary, M Abdel-Maaboud, M Mohamed, K Nasef. Minia University Hospital, Minia City, Egypt

Vitamin D insufficiency has been linked with susceptibility to infection, autoimmune diseases and cancers.

Objective To study the effect of vitamin D supplementation on the immunity and the risk of infections during the first year of life in full term infants.

Methods This is a prospective case control study included 99 full term infants attending Minia university hospital during the period from January 2010 to February 2012. They were divided into two groups: group I included forty-eight full term infants supplemented with daily 400 I.U vitamin D for 6-months after birth and group II included fifty-one full term infants not supplemented with vitamin D. History taking and clinical examination and other investigations needed for diagnosis of respiratory, GIT and urinary tract infections were addressed every visit for one year.

Results The incidences of infections totally were less common in infants supplemented with vitamin D than those not supplemented ( $p$-value $=0.01)$. Otitis media, bronchiolitis, pneumonia and gastroenteritis ( $\mathrm{p}$-value $=0.003,0.001,0.001$ and 0.01 respectively) were less common in them. There were no significant differences as regards urinary tract infections between the tow groups ( $p=0.3$ ). significant negative correlations between respiratory and GIT infections and the maternal vitamin $\mathrm{D}$ levels were present.

Conclusions Vitamin D supplementation decreased the incidence of infections especially respiratory and GIT infections. Maternal vitamin $\mathrm{D}$ levels correlated negatively with the incidence of infections.

\section{ROLE OF URINARY TRACT INFECTIONS IN NEONATAL INDIRECT HYPERBILIRUBINEMIA}

doi:10.1136/archdischild-2012-302724.0897

${ }^{1} \mathrm{E}$ Bahat Özdoğan, ${ }^{2} \mathrm{M}$ Mutlu, ${ }^{1} \mathrm{M}$ Kalyoncu, ${ }^{3} \mathrm{G}$ Karagüzel, ${ }^{2} \mathrm{Y}$ Aslan. ${ }^{1}$ Pediatric Nephrology; ${ }^{2}$ Neonatology; ${ }^{3}$ Pediatric Endocrinology, Karadeniz Technical University, Faculty of Medicine, Trabzon, Turkey
Urinary infections are an important cause of prolonged jaundice. But there is conflict about the role of the urinary infections on the pathological jaundice in the first 14 days of the life. This study aims to determine the frequency of urinary tract infections in neonates presenting with jaundice in the first 2 weeks of life with bilirubin levels that require phototherapy.

This study was done with neonates $2-14$ days old they have indirect bilirubin levels above the phototherapy limit but were not found to have any condition that would lead to elevated bilirubin levels, e.g. systemic infection, isoimmunization, erythrocyte enzyme defect, erythrocyte structural defect, hypothyroidism, sequestrated blood, polycythemia, or metabolic disease. Urine samples for urinalysis and culture were obtained using catheterization.

During the study, 482 neonates presented with jaundice and 262 of these fulfilled our criteria. UTI rate was $12 \%$. Mean bilirubin level was $20.9+6.1 \mathrm{mg} / \mathrm{dl}$. Thirtyfive $(13 \%)$ of these patients underwent to blood exchange, the rest were treated with phototherapy only. Weight loss in terms of percentage of birth weight was higher on uninfected patients and rebound bilirubin levels was higher on UTI group.

UTIs may present with isolated jaundice and may cause urosepsis, renal scarring, hypertension and chronic renal failure if they are not treated. In the neonatal period, infections lead to hyperbilirubinemia via hemolysis, inadequate conjugation, decreased excretion and oxidant stres. The findings of this study show the benefits of obtaining urine cultures for the diagnosis of UTI in neonatal patients with hyperibilirubinemia requiring phototherapy who have unexplained hyperbilirubinemia.

\section{REDUCING NEONATAL INFECTIONS IN SOUTH AND SOUTHERN CENTRAL VIETNAM: THE OPINION OF HEALTH CAREGIVERS}

doi:10.1136/archdischild-2012-302724.0898

'D Trevisanuto, 'V Dal Cengio, ' 2 Thi Ahn Thu, ${ }^{3} \mathrm{G}$ Arnolda, ${ }^{3} \mathrm{~T}$ Dihn Chien, ${ }^{3} \mathrm{D}$ Kumara, ${ }^{3} \mathrm{~L}$ Moccia, ${ }^{4} \mathrm{~N}$ Mihn Xuan. ${ }^{1}$ Children and Women's Health Department, Padua University School of Medicine, Padova, Italy; ${ }^{2}$ Cho Ray Hospital, Hochiminh City, Vietnam; ${ }^{3}$ East Meets West Foundation, Oakland, CA, USA; ${ }^{4}$ Tu Du Hospital, Hochiminh City, Vietnam

Background Infection represents a consistent cause of neonatal mortality in high as well as low income countries. In this study, we assessed the opinion of healthcare providers on how to improve neonatal infection control and prevention in South and Southern Central Vietnam hospitals.

Methods Fifty-four participants to a workshop on infection control and prevention were asked to fill out an anonymous, written questionnaire regarding the priorities that could improve neonatal infection control and prevention in provincial hospitals in South and Southern Central Vietnam.

Results Hand washing, exclusive breastfeeding and safe disposal of medical waste were scored by participants as the highest priorities for preventing neonatal infections. Education through instructional posters and written guidelines, family contact, kangaroo-mothercare, limitation of invasive procedures and screening for maternal GBS infection received relatively low scores.

Conclusions The opinions of operators involved in neonatal health match international recommendations for infection prevention with regards to some, but not all, issues, suggesting the need for a better understanding of this phenomenon. Our results may contribute to better design interventions for infection prevention in settings with limited resources.

\section{NEWBORNS AND INFECTIOUS RISKS:EXPERIENCE OF MATERNITY HOSPITAL SOUISSI-RABAT}

